\title{
LINEAR MAPS PRESERVING THE MINIMUM AND SURJECTIVITY MODULI OF OPERATORS
}

\author{
Mostafa MBeKHTA
}

Abstract. Let $\mathscr{B}(H)$ be the algebra of all bounded linear operators on a complex Hilbert space $H$, and denote by $\mathrm{m}(\mathrm{T})$ and $\mathrm{q}(\mathrm{T})$ respectively the minimum modulus and the surjectivity modulus for every $T \in \mathscr{B}(H)$. In this paper, we prove that if $\phi$ is a surjective unital linear map on $\mathscr{B}(H)$, then $\mathrm{m}(\phi(\mathrm{T}))=\mathrm{m}(\mathrm{T})$ for every $T \in \mathscr{B}(H)$ if and only if $\mathrm{q}(\phi(\mathrm{T}))=\mathrm{q}(\mathrm{T})$ for every $T \in \mathscr{B}(H)$ if and only if there exists an unitary operator $U \in \mathscr{B}(H)$ such that $\phi(T)=U T U^{*}$ for all $T \in \mathscr{B}(H)$.

Mathematics subject classification (2010): 47B48, 47A10, 46H05.

Keywords and phrases: Minimum modulus, surjectivity modulus, Jordan isomorphism, linear preservers.

\section{REFERENCES}

[1] B. Aupetit, A primer on spectral theory, Springer-Verlag, New York, 1991.

[2] B. Aupetit, Spectrum-preserving linear mappings between Banach algebras or Jordan-Banach algebras, J. London Math. Soc., 62 (2000), 917-924.

[3] B. Aupetit, Sur les transformations qui conservent le spectre, Banach Algebra'97 (Walter de Gruyter, Berlin), 1998, 55-78.

[4] M. BREŠAR AND P. ŠEMRL, Linear maps preserving the spectral radius, J. Funct. Anal., 142 (1996), $360-368$.

[5] M. BReŠAR AND P. ŠEMRL, Linear preservers on $\mathscr{B}(X)$, Banach Center Publications, 38 (1997), 49-58.

[6] M. BREŠAR AND P. ŠEMRL, Invertibility preserving maps preserve idempotents, Michigan Math. J., 45 (1998), 483-488.

[7] L.G. Brown And G.K. Pedersen, $C^{*}$-algebras of real rank zero, J. Funct. Anal., 99 (1991), 131 149.

[8] J. CuI, J. Hou, Linear maps between Banach algebras compressing certain spectral functions, Rocky Mountain J. Math., 34 (2004), 565-584.

[9] J. Dieudonné, Sur une généralisation du groupe orthogonal à quatre variables, Arch. Math (Basel), 1 (1949), 282-287.

[10] H.A. GINDLER AND A.E. TAYLOR, The minimun modulus of linear operator and its use in spectral theory, Studia Mathematica, 22 (1962), 15-41.

[11] A. GUTERMAN, C.-K. LI, AND P. ŠEMRL, Some general techniques on linear preserver problems, Linear Algebra Appl., 315 (2000), 61-81.

[12] I.N. Herstein, Jordan homomorphisms, Trans. Amer. Math. Soc., 81 (1956), 331-341.

[13] A. Jafarian AND A.R. Sourour, Spectrum preserving linear maps, J. Funct. Anal., 66 (1986), 255-261.

[14] I. KAPLANSKY, Algebraic and analytic aspects of operator algebras, Amer. Math. Soc., Providence, 1970.

[15] R. V. KADISON, Isometies of operator algebras, Annals of Mathematics, 54 (1951), 352-338.

[16] M. MarCUS AND R. PURVES, Linear transformations on algebras of matrices: The invariance of the elementary symmetric functions, Canad. J. Math., 11 (1959), 383-396. 
[17] M. MbekhtA, L. Rodman, AND P. ŠEMrL, Linear maps preserving generalized invertibility, Integral Equations Operator Theory, 55 (2006), 93-109.

[18] M. MвекhtA, Linear maps preserving a set of Fredholm operators, Proc. Amer. Math. Soc., to appear.

[19] V. MÜLLER, Spectral Theory of Linear Operators and Spectral Systems in Banach Algebras, Birkhäuser Verlag 2003.

[20] S. PierCe ET.AL., A survey of linear preserver problems, Linear and Multilinear Algebra, 33 (1992), $1-192$.

[21] A. PIETsCh, Operators Ideals, VEB Deutsch Verlag der Wissenschaften, Berlin, 1978.

[22] C.E. RickaRT, General Theory of Banach Algebras, Van Nostrand, Princeton, 1960.

[23] P. ŠEMRL, Two characterizations of automorphisms on $\mathscr{B}(X)$, Studia Mathematica, 105 (1993), $143-$ 149.

[24] A.R. Sourour, The Gleason-Kahane-Żelazko theorem and its generalizations, Banach Center Publications, 30 (1994), 327-331.

[25] A.R. Sourour, Invertibility preserving linear maps on $\mathscr{L}(X)$, Trans. Amer. Math. Soc., 348 (1996), $13-30$.

[26] J. Zemànek, Properties of the spectral radius in Banach algebras, Banach Center Publications, 8 (1982), 579-595. 